\title{
TRATAMIENTO PSICOLÓGICO EN EL TRASTORNO DE SOMATIZACIÓN: EFICACIA Y PROPUESTAS DE INTERVENCIÓN
}

\author{
JAVIER GARCÍA-CAMPAYO, AÍDA PASCUAL, \\ MARTA ALDA Y BÁRBARA OLIVÁN ${ }^{1}$
}

\author{
Hospital Universitario Miguel Servet y Universidad de Zaragoza \\ ${ }^{\mathrm{p}}$ Grupo Aragonés de Investigación en Atención Primaria
}

\begin{abstract}
Este trabajo de revisión analiza los estudios controlados randomizados realizados tanto en pacientes con trastorno de somatización como en pacientes con trastorno somatomorfo indiferenciado que han sido tratados mediante dos tipos de intervenciones: 1. Mediante la remisión de una carta de interconsulta por parte de un especialista de salud mental a atención primaria y 2. mediante terapia cognitivo-conductual. En estos estudios se analizan variables como el entorno, el contenido de la terapia, el formato, el número de sesiones, las variables de resultado, el tiempo de seguimiento, etc. Se resumen las principales conclusiones de las intervenciones psicológicas realizadas hasta el momento en estos pacientes y se realizan propuestas para nuevos terapias cognitivo-conductuales en escalado a realizar en el futuro en pacientes con trastorno de somatización.
\end{abstract}

Palabras clave: Trastorno de somatización, trastorno somatomorfo indiferenciado, terapia cognitivo-conductual.

\section{Psychological treatment in somatization disorder: effectiveness and intervention proposals}

This review analyze randomized controlled studies carried out both in somatization disorder and in undifferentiated somatoform disorder patients that had been treated using two kind of interventions: 1 . A consultation letter referred by a mental health specialist to the patient's family doctor and 2. Using cognitive-behaviour therapy. In these studies some variables such as the setting, the content, the number of sessions, the outcome, the follow-up, etc. has been assessed. We summarize the main conclusions on psychological interventions developed up to the moment in these patients and we propose new stepped cognitive-behavioural therapies to implement in the future in somatization disorder patients.

Key words. Somatization disorder, undifferentiated somatoform disorder, cognitivebehaviour therapy.

\section{EL TRASTORNO DE SOMATIZACIÓN}

El trastorno de somatización se define como una enfermedad consistente en una historia de síntomas físicos no explicados médicamente que ocurren durante un período de varios años (APA, 2000; WHO, 1992). Según el DSM-IV-TR el inicio de la enfermedad tiene que ser previo a la edad de 30 años, y los síntomas tie-

Correspondencia: Javier García-Campayo, Avda Gómez Laguna 52, 4. D, 50009 Zaragoza. Correo-e: jgarcamp@arrakis.es nen que incluir al menos cuatro síntomas de dolor, dos síntomas gastrointestinales, uno sexual y uno pseudoneurológico o de conversión (APA, 2000). Los estudios epidemiológicos que utilizan los criterios diagnósticos del DSM-III describen tasas de prevalencia de aproximadamente 1$1,4 \%$ en atención primaria (Kirmayer y

\footnotetext{
Agradecimientos: Este trabajo ha sido posible gracias a la Red de Investigación en Actividades de Prevención y Promoción de la Salud (REDIAPP-G03/170) y a la Red Temática de Investigación Cooperativa «Psiquiatría de Enlace» (N. ${ }^{\circ}$ Expediente: G03/128) del Instituto de Salud Carlos III de Madrid.
} 
Robbins, 1991; Simon y Gureje, 1999) y de entre el 0,04 y el $0,4 \%$ en la población general (Swartz et al., 1986).

Los síntomas más frecuentes en el trastorno de somatización son dolor en diferentes partes del cuerpo (cabeza, tórax, espalda, articulaciones, pelvis), así como disfagia, náuseas, estreñimiento, distensión abdominal, palpitaciones, vértigos y dificultad para respirar (Liu et al., 1997). Los síntomas somáticos se asocian, frecuentemente, a trastornos de ansiedad y depresión comórbidos (Swartz et al., 1986). Los pacientes con trastornos de somatización son grandes utilizadores del sistema sanitario incluyendo consultas médicas, hospitalizaciones, procedimientos quirúrgicos y pruebas complementarias (Smith, 1994).

\section{TRATAMIENTO DEL TRASTORNO DE SOMATIZACIÓN A NIVEL DE ATENCIÓN PRIMARIA}

En muchas enfermedades psiquiátricas, una de las preguntas clave respecto al tratamiento es en qué nivel del sistema sanitario es necesario abordar al paciente. Para ello es necesario conocer los caminos que siguen los enfermos para solicitar ayuda médica (García-Campayo et al., 1997). En países que poseen un sistema público y universal como el National Health Service británico o el sistema sanitario español, los pacientes somatizadores acuden al médico de familia, que es la puerta de entrada al sistema de salud y, una vez allí, se crea un circuito en forma de pelota de pingpong entre las especialidades médicoquirúrgicas y el médico generalista. Si, ocasionalmente, el paciente acude a salud mental, lo más probable es que sea remitido de nuevo a esta vorágine con un informe donde conste que no existe una enfermedad mental. En este peregrinar del somatizador llama la atención el amplísimo uso de las medicinas alternativas (García Campayo y Sanz Carrillo, 2000). En lugares donde el sistema de salud se encuentra más privatizado, por ejemplo en los Estados Unidos, el médico de familia no siempre es la puerta de acceso al sistema ya que el paciente puede elegir directamente al especialista. Esto, en el caso concreto de los somatizadores, es perjudicial porque: (a) carecen de un médico de referencia que les conozca y en quien confiar, y (2) la práctica privada con elección directa de especialista favorece el uso masivo de pruebas complementarias con lo cual se refuerza la conducta de enfermedad del paciente y su cronificación.

Dentro del sistema sanitario, la atención primaria es especialmente importante porque constituye el primer contacto del somatizador con el sistema sanitario, y porque la mayor parte de los pacientes son tratados a este nivel, por lo que es en este entorno donde más posibilidades de éxito tiene cualquier intervención (García-Campayo et al., 1997). Existen unas normas de buena práctica clínica con los pacientes con trastorno de somatización en atención primaria, que ha propuesto el grupo de Smith (Smith, 1991; Smith et al., 1995) y que se consideran de referencia. Las resumimos en la Tabla 1.

Estas recomendaciones se han demostrado efectivas obteniéndose un ahorro de los gastos sanitarios producidos por los somatizadores de un 32\% (Smith et al., 1995). La mayor dificultad para llevar a cabo estos programas es que los somatizadores crónicos producen sentimientos de frustración e ira y provocan un gran rechazo por parte de los profesionales sanitarios. De hecho, cuando se realizan encuestas a los médicos de familia, el $70 \%$ rechaza ofrecer cualquier tipo de apoyo psicológico a los somatizadores aunque se les entrene especificamente para ello (García-Campayo et al., 1998; 1999; Sanz Carrillo y García-Campayo, 
Tabla 1. Normas de buena práctica clínica en atención primaria con los pacientes

con trastornos de somatización

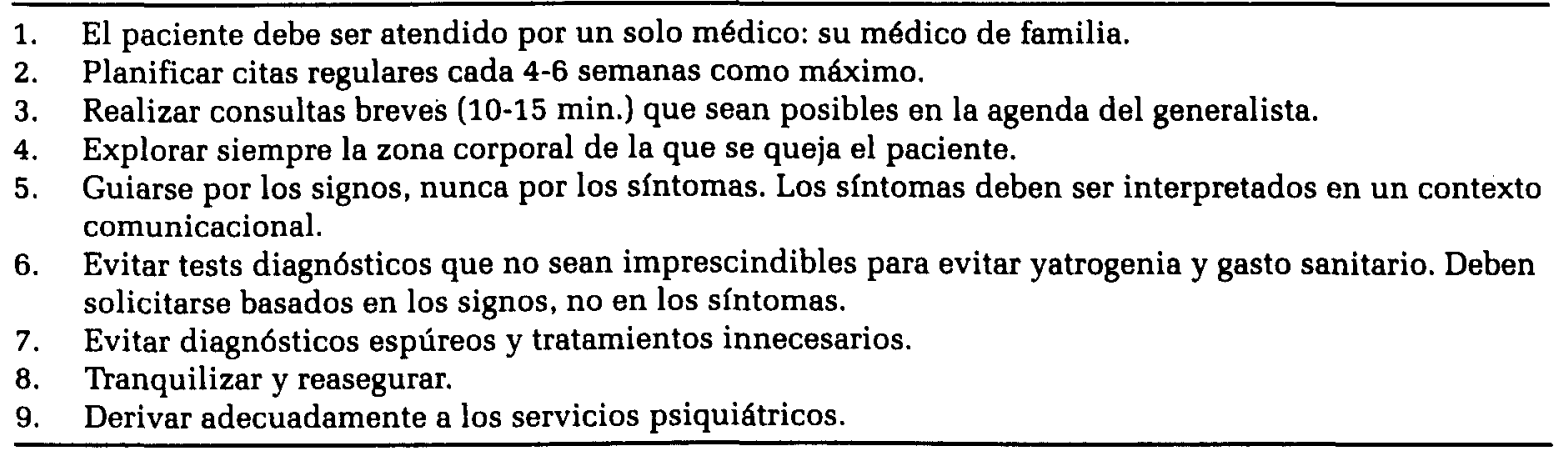

1999). Por eso, se considera imprescindible la creación de unidades de interconsulta en somatización en atención primaria con tres funciones básicas: formación de los profesionales, enlace para diseñar estrategias de actuación con estos enfermos e investigación (García-Campayo et al., 1997).

\section{ESTUDIOS CONTROLADOS SOBRE EL TRATAMIENTO PSICOLÓGICO DEL TRASTORNO DE SOMATIZACIÓN}

Los trabajos de investigación mediante estudios controlados randomizados que han evaluado la eficacia de las intervenciones psicológicas en el tratamiento de los pacientes con trastorno de somatización se agrupan en dos según el tipo de intervención sea: a) una carta de interconsulta remitida por el especialista de salud mental al médico de familia, o b) terapia cognitivo-conductual.

\section{a) Cartas de interconsulta}

Se han desarrollado dos estudios controlados randomizados evaluando la utilidad de enviar una carta de interconsulta a para informar/entrenar al medico de familia que solicita consulta sobre el tratamiento más adecuado para este tipo de pacientes (Rost et al., 1994; Smith et al.,
1986). En el primero de ellos (Smith et al., 1986) se randomizan 41 pacientes con diagnóstico DSM-III de trastorno de somatización diagnosticados mediante entrevista psiquiátrica estructurada, que fueron distribuidos en dos grupos: lista de espera o un grupo en el que los generalistas recibieron una carta de consulta donde un especialista recomendaba una serie de intervenciones a realizar con estos pacientes. Los pacientes fueron seguidos durante 3 meses por un investigador ciego que desconocía a qué grupo pertenecía el paciente. La valoración del paciente incluía, aparte del uso de servicios sanitarios, su situación física y psicológica evaluada mediante el Rand Health Status Measures (RAND). Los pacientes pertenecientes al grupo que recibieron la carta de interconsulta disminuyeron el gasto sanitario en un 53\% sobre todo por disminución de los ingresos hospitalarios. No se observaron cambios en el estado físico o psicológico en ninguno de los dos grupos (Smith et al., 1986) (ver Tabla 2).

El segundo estudio fue una replicación del trabajo anterior ampliando el tamaño muestral a 73 pacientes realizada por Rost et al. (1994) también en pacientes con trastorno de somatización según criterios DSM-III-R mediante entrevista psiquiátrica. En este estudio, los pacientes sobre los que se realiza la intervención 
Tabla 2. Estudios controlados randomizados mediante cartas de interconsulta en el tratamiento del trastorno de somatización

\begin{tabular}{|c|c|c|c|c|c|c|}
\hline Autor & $N$ & & $\begin{array}{l}\text { Intervención y } \\
\text { grupo control }\end{array}$ & $\begin{array}{c}\text { Medidas / } \\
\text { Evaluadores }\end{array}$ & $\begin{array}{c}\text { Meses } \\
\text { seguimiento }\end{array}$ & Resultados \\
\hline Smith et al., 1986 & 41 & $\begin{array}{l}1 . \\
2 .\end{array}$ & $\begin{array}{l}\text { Visitas regulares, } \\
\text { intervenciones } \\
\text { limitadas. } \\
\text { No se realizan } \\
\text { visitas }\end{array}$ & $\begin{array}{l}\text { Autorreportadas. } \\
\text { Los recolectores de } \\
\text { datos son ciegos }\end{array}$ & 18 & $\begin{array}{l}53 \% \text { disminución } \\
\text { costes de salud. } \\
\text { No cambios salud } \\
\text { física o mental ni } \\
\text { en grupo de } \\
\text { intervención ni en } \\
\text { control }\end{array}$ \\
\hline Rost et al., 1994 & 73 & $\begin{array}{l}1 . \\
2 .\end{array}$ & $\begin{array}{l}\text { Visitas regulares, } \\
\text { intervenciones } \\
\text { limitadas. } \\
\text { No se realizan } \\
\text { visitas }\end{array}$ & $\begin{array}{l}\text { Autorreportadas. } \\
\text { Los recolectores de } \\
\text { datos son ciegos }\end{array}$ & 18 & $\begin{array}{l}\text { En relación al } \\
\text { grupo control: } \\
\text { Disminución } \\
21 \% \text { costes de } \\
\text { salud, no mejora } \\
\text { función, física, no } \\
\text { hay cambios en } \\
\text { salud mental }\end{array}$ \\
\hline
\end{tabular}

mediante la carta de consulta, disminuyen también el gasto sanitario pero en menor medida: un $21 \%$. En este caso, mejoran algo la función física pero, de nuevo, no mejora en absoluto su salud psicológica (Tabla 2). En resumen, las cartas de consulta realizadas por los especialistas al médico de familia son útiles en la reducción de costes en todos los casos, en algunos casos pueden mejorar la función física, pero en ningún caso mejoran el bienestar psicológico. La razón podría ser que es una intervención no dirigida al paciente sino que el objeto de intervención es el propio médico.

\section{b) Terapia cognitivo-conductual}

Existen otros dos estudios controlados randomizados en pacientes con trastorno de somatización utilizando esta técnica psicológica. Un primer estudio, realizado por Kashner et al. (1995) en una muestra de 70 sujetos, comparaba pacientes sobre los que no se realizaba tratamiento con otros a los que se les ofrecía 8 sesiones de terapia de grupo en las que se incluía resolución de proble- mas, afrontamiento, asertividad y manejo de la expresión emocional. Al menos 22 de los 44 pacientes faltaron a alguna de las sesiones. En el análisis por inten- ción de tratar se comprobó que los pacientes randomizados en el grupo de terapia habían consumido un $27 \%$ menos de recursos sanitarios y además habían mejorado tanto en su función física como en la psicológica medida mediante el cuestionario RAND. Existía correlación positiva entre asistencia a las sesiones de terapia y mejora en la función física y psicológica (ver Tabla 3).

El segundo estudio, realizado por Lidbeck (1977) en una muestra de 50 sujetos, se realizó también sobre pacientes con trastorno de somatización pero utilizando criterios diagnóstico menos estrictos, los del Comité de Clasificación de la Wonca (Classification Comittee of Wonca, 1983). La terapia grupal incluía educación sobre la enfermedad, técnicas de relajación, habilidades de afrontamiento y resolución de problemas. El efecto de la terapia de grupo fue de 0,67 al final del tratamiento y de 0,8 seis meses más tarde, ambos medidos por el Illness Beha- 
Tabla 3. Estudios controlados randomizados mediante terapia cognitivo conductual en el tratamiento del trastorno de somatización

\begin{tabular}{|c|c|c|c|c|c|c|}
\hline Autor & $N$ & & $\begin{array}{l}\text { Intervención y } \\
\text { grupo control }\end{array}$ & $\begin{array}{c}\text { Medidas / } \\
\text { Evaluadores }\end{array}$ & $\begin{array}{c}\text { Meses } \\
\text { seguimiento }\end{array}$ & Resultados \\
\hline Kashner et al., 1997 & 70 & 2. & $\begin{array}{l}\text { Carta de consulta, } \\
\text { terapia de grupo, } \\
\text { resolución de } \\
\text { problemas, } \\
\text { habilidades de } \\
\text { afrontamiento. } \\
\text { Carta de consulta. }\end{array}$ & $\begin{array}{l}\text { Autorreportada / } \\
\text { Recolección de } \\
\text { datos ciega. }\end{array}$ & 12 & $\begin{array}{l}\text { Intervención en } \\
\text { relación al grupo } \\
\text { por intención de } \\
\text { tratar: reduce } \\
\text { gastos sanitarios } \\
31 \% \text {, mejora } \\
\text { salud mental y } \\
\text { función física. }\end{array}$ \\
\hline Lidbeck et al., 1997 & 50 & 2. & $\begin{array}{l}\text { TCC, educación, } \\
\text { relajación, } \\
\text { habilidades de } \\
\text { afrontamiento, } \\
\text { resolución de } \\
\text { problemas. } \\
\text { Lista de espera. }\end{array}$ & Autorreportado. & 6 & $\begin{array}{l}\text { Diferencia media } \\
\text { estandarizada } \\
\text { (IC } 95 \%) \text { : } \\
\text { Postratamiento: } \\
0,67(0,07-1,27) \\
\text { Seguimiento: } \\
0,80(0,19-1,42)\end{array}$ \\
\hline
\end{tabular}

viour Questionnaire (Bradley et al., 1992) (ver Tabla 3). Sin embargo, no se objetivó ningún cambio en síntomas de ansiedad o depresión. En ambos estudios, aunque las terapias están bien descritas, no se encuentran manualizadas y no existen procedimientos para asegurar que se están aplicando de forma consistente las técnicas propuestas.

\section{APORTACIONES DE OTROS ESTUDIOS REALIZADOS EN PACIENTES CON TRASTORNO SOMATOMORFO INDIFERENCIADO}

Los trastornos somatomorfos indiferenciados constituyen una patología mucho más prevalente en la práctica clínica que el trastorno de somatización (Katon y Walter, 1998), ya que el DSM-IV-TR sólo exige uno o más síntomas físicos no explicados médicamente cuya duración sea superior a 6 meses y que produzca malestar o incapacidad. Dada su mayor prevalencia se han realizado un gran número de ensayos clínicos controlados randomizados evaluando la eficacia de los trata- mientos psicológicos en estos pacientes y existen excelentes revisiones sobre este tema (Kroenke y Swindle, 2000). Sin embargo, la mayor parte de los estudios tienden a agrupar a los pacientes según el síntoma predominante, conformando categorías diagnósticas dentro de la especialidad médica que agrupa los síntomas físicos no explicados médicamente. En la Tabla 4 resumimos las principales enfermedades que pueden incluirse como trastorno somatomorfo indiferenciado en las diferentes especialidades médicas.

La mayoría de los estudios se han realizado en fibromialgia, fatiga crónica, dolor de espalda, dolor torácico atípico, tinnitus y boca dolorosa. Sólo existen 5 estudios controlados randomizados realizados en pacientes diagnosticados de trastorno somatomorfo indiferenciado en que los pacientes sean clasificados como tal y que, por tanto, sus conclusiones son extrapolables a las de los pacientes con trastorno de somatización.

En uno de ellos se utilizó una carta de interconsulta (Smith et al., 1995) y se consiguió una reducción en los costes del $33 \%$ pero no mejoró la función física ni 
Tabla 4. Síndromes funcionales, por especialidades, que se incluyen en la categoría de trastorno somatomorfo indiferenciado

\begin{tabular}{ll}
\hline Especialidad & Síndromes \\
\hline Cardiología & Dolor torácico atípico \\
Digestivo & Disfagia, síndrome del intestino irritable, dispepsia no ulcerosa \\
Respiratorio & Asma funcional \\
Neurología & Cefalea funcional, síncope, vértigo funcional \\
Reumatología & Fibromialgia, síndrome de dolor miofascial \\
Infeccioso & Fatiga crónica, sensibilidad ambiental, síndrome de alergia total \\
ORL & Boca dolorosa, tinnitus \\
Ginecología & Dolor pélvico, dismenorrea y dispareunia funcionales, vaginismo, vulvodinia, sín- \\
& drome tensión premenstrual \\
Urología & Cistitis intersticial \\
\hline
\end{tabular}

la psicológica. En otros 3 se empleó terapia cognitivo conductual individual: En el primero (Speckens et al., 1995) mejoró la conducta de enfermedad medida con el Illness Behaviour Questionnaire a los 6 meses pero no a los 12 meses y no mejoró la ansiedad ni la depresión a los 6 meses. El segundo (McLeod et al., 1997) mostró clara mejoría en ansiedad y depresión medida con el SCLR-90 al final del tratamiento que se mantenía a los 6 meses. Sin embargo, mostraba graves problemas metodológicos: en el grupo de intervención hubo un gran número de abandonos que no se contabilizaron en el análisis. El tercer estudio (Sumathipala et al., 2000) mostró una importante mejoría en el malestar general, los síntomas físicos y el número de visitas al sistema sanitario al final del tratamiento, pero no se realizó estudio de seguimiento. Existe un último estudio en el que se empleó terapia cognitivo conductual en formato grupal asociada a meditación y relajación (Hellman et al., 1990). Este estudio mostró una mejora significativa en síntomas somáticos, malestar psicológico y visitas al médico tanto al final del tratamiento como a los 6 meses de seguimiento.

Las conclusiones que podemos deducir de los estudios de intervención psicológica realizados hasta este momento en pacientes con síntomas somáticos los resumimos en la Tabla 5.
RECOMENDACIONES PARA FUTUROS ESTUDIOS DE INTERVENCIÓN PSICOLÓGICA EN PACIENTES CON TRASTORNO DE SOMATIZACIÓN

Actualmente no existe suficiente evidencia científica como para recomendar un tratamiento psicológico específico en el trastornos de somatización, como se recoge en los manuales de evidencia clínica (Centro Cochrane Iberoamericano, 2004). En base a los estudios existentes sobre el tema, las intervenciones psicológicas que se desarrollen en el futuro para estos pacientes deberían poseer las siguientes características:

1. La intervención se debe realizar a nivel de atención primaria, puerta de entrada de los pacientes al sistema, y donde antes y mejor puede intervenirse para evitarse la cronificación.

2. La intervención debe poseer un sistema en escalado («stepped») de forma que se realicen intervenciones más sencillas en los casos más leves que vayan aumentando en complejidad, en cuanto a especialización de los profesionales y tiempo invertido, en los casos más resistentes, para optimizar el coste/beneficio del tratamiento.

3. En base a lo que sabemos sobre la eficacia de las intervenciones psicológi- 
Tabla 5. Conclusiones de los estudios de intervención psicológica realizados en pacientes con trastorno de somatización y trastorno de somatización indiferenciado

a) ENTORNO:

1. Las intervenciones psicológicas han sido realizadas en salud mental y NO HAY ESTUDIOS EN ATENCIÓN PRIMARIA. Por lo tanto, la eficacia en este entono de la psicoterapia (la terapia cognitivo conductual sería la más utilizada en este grupo de trastornos) aún debe ser evaluada.

2. NO SE HAN REALIZADO INTERVENCIONES EN ESCALADO (STEPPED) PARA DIFERENTES NIVELES DE GRAVEDAD: Es decir, intervenciones sencillas para pacientes leves (por ejemplo, cartas de interconsulta para el médico de familia), y otras más complejas (por ejemplo psicoterapia cognitivoconductual) para pacientes más graves. Esto es clave para la reducción de costes.

3. NO SE HAN REALIZADO INTERVENCIONES ESPECfFICAS dirigidas a la comorbilidad psicológica en el Eje I del trastorno de somatización (trastornos de depresión, ansiedad, etc.).

\section{b) CONTENIDO:}

4. Las intervenciones tienden a ser MULTIFACÉTICAS con un énfasis variable en los aspectos cognitivos, conductuales y de relajación.

5. Los formatos tienden a ser FLEXIBLES y no estrictamente manualizados (aunque esto contradice las recomendaciones habituales en los trabajos de investigación en psicoterapia) (Aveline y Shapiro 1995).

6. Aunque parece que la somatización puede ser REFORMULADA en términos psicológicos (o reatribuida), por lo menos para aquellos pacientes que desean recibir terapia psicológica, no está claro qué porcentaje de las pérdidas de los pacientes a los que se les oferta tratamiento estarían causadas por no aceptar la causa psicológica de sus síntomas

c) FORMATO/ADMINISTRACIÓN:

7. Tanto los formatos grupales como los individuales pueden ser efectivos.

8. Son efectivas tanto un numero de sesiones fija como un numero de sesiones flexible adaptada a las necesidades del paciente.

9. Aunque el número de sesiones varía, se ha demostrado que son EFECTIVAS terapias tan breves como las de 5 sesiones (esta parece ser la dosis mínima efectiva).

d) EFICACIA:

10. Se han usado varios grupos control como comparadores. El grupo de control más conservador ha sido el de terapia conductual (operante o de relajación).

11. El tiempo de seguimiento suele ser razonable en todos los estudios pero en ningún caso excede los 12 meses. No se han estudiado los efectos más allá de dos años. En algunos estudios se ha demostrado efecto de mantenimiento: la eficacia de la terapia continua incluso después de haber terminado su administración.

12. LA MEDIDA DE RESULTADO QUE MEJOR EVOLUCIONA SON LOS COSTES, con una media de ahorro de gastos sanitarios respecto al grupo control entre el $20 \%$ y el $50 \%$.

13. DENTRO DE LOS SÍNTOMAS DEL PACIENTE, LOS SINTTOMAS SOMÁTICOS SON LOS QUE MEJOR EVOLUCIONAN independientemente del resto de parámetros psicológicos. De hecho, los resultados sobre ansiedad y depresión son poco satisfactorios.

cas en estos pacientes, estas debieran ser:

- Multimodales: Con predominio de abordajes cognitivo-conductuales pero incluyendo aspectos como relajación, resolución de problemas, educación y afrontamiento.

- Grupales: Es una modalidad que maximiza el coste/beneficio y que se ha mostrado eficaz en los estudios previos en estos pacientes. 
- Breves: Se ha demostrado que a partir de 5 sesiones pueden ser efectivas. Las perdidas a partir de la 6-8 sesión superan el $30 \%$ y la eficacia del tratamiento correlaciona con la asistencia a la terapia (Kashner et al., 1995). Se considera una terapia estándar 8 sesiones grupales de 2 horas.

4. El seguimiento debiera realizarse al menos durante 2 años, dada la tendencia de esta enfermedad a presentar empeoramientos y mejorías en la evolución habitual que pueden artefactar los resultados del estudio.

5. No hay comparaciones transculturales sobre la eficacia de las intervenciones psicológicas en esta enfermedad, pese a conocerse que los trastornos somatomorfos, junto a los trastornos de personalidad, constituyen algunos de los apartados de las clasificaciones psiquiátricos más claramente influenciados por la cultura (Mezzich et al., 1996).

\section{CONCLUSIONES}

Los trastornos de somatización constituyen una patología importante para el sistema sanitario de los países desarrollados por el enorme coste sanitario que suponen (Smith, 1994). Sin embargo, y hasta el momento, no existen suficientes evidencias científicas para recomendar ningún tratamiento psicológico o farmacológico específico en estos pacientes y, especialmente, en el trastorno de somatización.

Sobre las bases de los datos resumidos en este artículo se está implementando un estudio multicéntrico internacional de intervención psicológica en escalado en pacientes con trastorno de somatización diagnosticados en atención primaria que se va a desarrollar en tres países:
Estados Unidos (Prof. Escobar, Universidad de New Jersey), Alemania (Prof. Rief, Universidad de Groningen) y España (los autores del artículo en la Universidad de Zaragoza). Esperamos que este sea el punto de partida para desarrollar la evidencia científica suficiente como para poder recomendar una intervención psicológica específica en pacientes con trastorno de somatización.

\section{REFERENCIAS BIBLIOGRÁFICAS}

American Psychiatric Association (2000). Diagnostic and statistical manual of mental disorders. (Text revised). Washington, DC: APA.

Aveline, M., y Shapiro, D.A. (Eds.) (1995). Research foundations for psychotherapy practice. Chichester: John Wiley \& Sons.

Bradley, L.A., McDonald, H.J., y Jaworski, T.M. (1992). Assessment of psychological status using interviews and self-report instruments. En D.C. Turk y R. Melzack (Eds.), Handbook of pain assessment. (pp. 193213). New York: Guilford.

Centro Cochrane Iberoamericano (2004). Evidencia clínica concisa. Bogotá: Legis.

Classification Committee of Wonca in Collaboration with the World Health Organization (1983). ICHPPC-2-Defined (International Classification of Health Problems in Primary Care. Oxford: United Kingdom: Oxford University Press.

García-Campayo, J., Sanz Carrillo, C., Yoldi, A., y López Aylon, R. (1998). Management of somatizers in primary care: are family doctors motivated? Australian and New Zealand Journal of Psychiatry, 32, 528-533.

García-Campayo, J., y Sanz-Carrillo, C. (2000). The use of alternative medicines by somatoform disorder patients in Spain. British Journal of General Practice, 50, 487-488.

García-Campayo, J., Sanz-Carrillo, C., Claraco, L.M., Arana, A., y Montón, C. (1997). The challenge of somatization - the need for liaison units: mental health in primary care. Primary Care Psychiatry, 3, 163-169. 
García-Campayo, J., Sanz-Carrillo, C., y Montón, C. (1999). Dificultades en la relación médico-paciente en somatizadores (I): expectativas del paciente. Medicina Clínica (Barcelona), 112, 102-105.

Hellman, C.J., Budd, M., Borysenko, J., McClelland, D.C., y Benson, H. (1990). A study of the effectiveness of two group behavioral medicine interventions for patients with psychosomatic complaints. Behavioral Medicine, 16, 165-173.

Kashner, T.M., Rost, K., Cohen, B., Anderson, M., y Smoth, G.R.Jr. (1995). Enhancing the health of somatization disorder patients: effectiveness of short-term group therapy. Psychosomatics, 36, 462-470.

Katon, W.J., y Walker, E.A. (1998). Medically unexplained symptoms in primary care. Journal of Clinical Psychiatry, 59, 15-21.

Kirmayer, L.J., y Robbins, J.M. (1991). Three forms of somatization in primary care: prevalence, co-occurrence and socio-demographic characteristics. Journal of Nervous and Mental Disorders, 179, 647-655.

Kroenke, K., y Swindle, R (2000). Cognitivebehavioral therapy for somatization and symptoms syndromes: A critical review of controlled critical trials. Psychotherapy and Psychosomatics, 69, 205-215.

Lidbeck, J. (1997). Group therapy for somatization disorder in general practice: effectiveness of a short cognitive-behavioural treatment model. Acta Psychiatrica Scandinavica, 96, 14-24.

Liu, G., Clark, M.R., y Eaton, W.W. (1997). Structural factor analysis for medically unexplained somatic symptoms of somatization disorder in the Epidemiological Catchment Area Study. Psychological Medicine, 278, 617-626.

McLeod, C.C., Budd, M.A., y McClelland, D.C (1997). Treatment of somatization in primary care. General Hospital Psychiatry, 19, 251-258.

Mezzich, J.E., Kleinman, A., Fabrega, H.Jr., y Parron, D.L. (1996). Culture \& Psychiatric diagnosis. A DSM-IV perspective. Washington: American Psychiatric Press.

Rost, K., Kashner, T.M., y Smith, G.R.Jr. (1994). Effectiveness of psychiatric intervention with somatization disorder patients: improved outcomes at reduced costs. General Hospital Psychiatry, 16, 381387.

Sanz-Carrillo, C., García-Campayo, J., y Montón C. (1999). Dificultades en la relación médico-paciente en somatizadores (II): reacciones del profesional y tipos de relación. Medicina Clínica (Barcelona) 112, 147-150.

Simon, G.E., y Gureje, O (1999). Stability of somatization disorder and somatization symptoms among primary care patients. Archives of General Psychiatry, 56, 90-95.

Smith, G.R (1991). Somatization disorder in the medical setting. Washington: American Psychiatric Press.

Smith, G.R.Jr. (1994). The course of somatization and its effect on utilization on health care resources. Psychosomatics, 35, 263-267.

Smith, G.R.Jr., Monson, R.A., y Debby, C.R (1986). Psychiatric consultation in somatization disorder: a randomized controlled study. New England Journal of Medicine, 314, 1407-1413.

Smith, G.R.Jr., Rost, K., y Kashner, M. (1995). A trial of the effect of a standardized psychiatric consultation on health outcomes and costs in somatizing patients. Archives of General Psychiatry, 52, 238-243.

Speckens, A.E.M., van Hemert, A.M., Spinhoven, P., Hawton, K.E., Bolk, J.H., y Rooijmans, H.G.M. (1995). Cognitive behavioral therapy for medically unexplained physical symptoms: a randomized controlled trial. British Medical Journal, 311, 1328-1832.

Sumathipala, A., Hewege, S., Hanwella, R., y Mann, A.H. (2000). Randomized controlled trial of cognitive behavior therapy for repeated consultations for medically unexplained complaints: a feasibility study in Sri Lanka. Psychological Medicine, 3, 747-757.

Swartz, M., Blazer, D., George, L., y Landerman, R. (1986). Somatization disorder in a community population. American Journal of Psychiatry, 143, 1403-1408.

World Health Organization (1992). The ICD10 Classification of mental and behavioural disorders: Clinical descriptions and diagnostic guidelines. Géneva: Switzerland. 